ESJ Social Sciences

\title{
Análisis Estadístico De La Formación Digital A Partir De La Relación De La Desigualdad Ingresos (Coeficiente Gini) Y El Analfabetismo Digital En Ecuador
}

\author{
Albán Defilippi María Teresa \\ Licenciada en Ciencias de la Educación Mención Lengua Inglesa y Lingüística, Magister en \\ Pedagogía de los Idiomas Nacionales y Extranjeros Mención en Enseñanza de Inglés \\ Docente Universidad de Guayaquil \\ Córdova Pozo Mónica Lissette \\ Licenciada en Ciencias de la Educación Mención Comercio Exterior \\ Correa Verduga Gema Carolina \\ Licenciada en Ciencias de la Educación mención Educación Parvularia, Maestra en \\ Administración de la Educación \\ Falconí Orozco Guisela Mariuxi \\ Secretaria Ejecutiva en Archivo y Sistemas de Información, Ingeniera Comercial, Magister \\ en Administración de Pequeñas y Medianas Empresas, Master Universitario en Liderazgo y \\ Dirección de Centros Educativos
}

\section{Mosquera Moreno Gladys Elizabeth \\ Ingeniera Comercial}

Doi:10.19044/esj.2021.v17n8p10

Submitted: 11 February 2021

Accepted: 09 March 2021

Published: 31 March 2021
Copyright 2021 Author(s)

Under Creative Commons BY-NC-ND

4.0 OPEN ACCES

Cite As:

Alban MT., Cordova, PML., Correa, VGC., Falconi, OGM., and Mosquera, MGE., (2021). Análisis Estadístico De La Formación Digital A Partir De La Relación De La Desigualdad Ingresos (Coeficiente Gini) Y El Analfabetismo Digital En Ecuador. European Scientific Journal, ESJ, 17(8), 10. https://doi.org/10.19044/esj.2021.v17n8p10

\section{Resumen:}

El estudio se basa en los efectos provocados por el analfabetismo digital desde la perspectiva de los ingresos, se puede entender en la oportunidad de conseguir un trabajo adecuado que aporte al bienestar de la ciudadanía. Ecuador en comparación con otros países de América Latina y el mundo, presenta una baja conectividad y desarrollo laboral por competencias enfocadas en la práctica de uso de TICs. Por lo consiguiente, esta 
investigación tiene como objetivo analizar la relación que existe entre Desigualdad Ingresos (Coeficiente Gini) y el analfabetismo digital (Porcentaje personas) basándose en datos específicos de Ecuador entre los años 2008 - 2019. La metodología aplicada fue un enfoque cuantitativo, con alcance descriptivo y diseño no experimental transeccional. La técnica de recolección de información fue estadística y se aplicó el modelo de regresión lineal simple basado en variables analizadas en los estudios teóricos como los de Katz \& Koutroumpis (2012) y de Galperin y Ruzzier (2010). Los resultados fueron que existe suficiente evidencia estadística para demostrar la relación entre la variable dependiente $\mathrm{Y}=$ Analfabetismo digital (Porcentaje de personas) y la variable independiente $\mathrm{X}=$ Desigualdad Ingresos (Coeficiente Gini) con una significación ANOVA de $0.00 \%$, un $\mathrm{R}^{\wedge} 2=86.3 \%$, del cual indica que el modelo tiene un buen ajuste a la variable explicativa, con una sensibilidad de $\beta=0.438$.

Palabras claves: Analfabetismo digital, Desigualdad de ingresos, TICs, Brecha digital, Competencias laborables. 


\title{
Statistical Analysis Of Digital Training Based On The Relation of Income Inequality (Gini Coefficient) And Digital Illiteracy in Ecuador
}

\author{
Albán Defilippi María Teresa \\ Licenciada en Ciencias de la Educación Mención Lengua Inglesa y Lingüística, Magister en \\ Pedagogía de los Idiomas Nacionales y Extranjeros Mención en Enseñanza de Inglés \\ Docente Universidad de Guayaquil \\ Córdova Pozo Mónica Lissette \\ Licenciada en Ciencias de la Educación Mención Comercio Exterior \\ Correa Verduga Gema Carolina \\ Licenciada en Ciencias de la Educación mención Educación Parvularia, Maestra en \\ Administración de la Educación

\section{Falconí Orozco Guisela Mariuxi} \\ Secretaria Ejecutiva en Archivo y Sistemas de Información, Ingeniera Comercial, Magister \\ en Administración de Pequeñas y Medianas Empresas, Master Universitario en Liderazgo y \\ Dirección de Centros Educativos
}

\section{Mosquera Moreno Gladys Elizabeth \\ Ingeniera Comercial}

\begin{abstract}
:
The study is based on the effects caused by digital illiteracy from the perspective of income, it can be understood in the opportunity to get a suitable job that contributes to the well-being of citizens. Ecuador compared to other countries in Latin America and the world, has low connectivity and job development due to competencies focused on the practice of using ICTs. Therefore, this research aims to analyze the relationship between Income Inequality (Gini Coefficient) and digital illiteracy (Percentage of people) based on specific data from Ecuador between the years 2008 - 2019. The applied methodology was a quantitative approach, with descriptive scope and non-experimental transectional design. The information collection technique was statistical, and the simple linear regression model was applied based on variables analyzed in theoretical studies such as those of Katz \& Koutroumpis (2012) and Galperin and Ruzzier (2010). The results were that there is enough statistical evidence to demonstrate the relationship between the dependent variable $\mathrm{Y}=$ Digital illiteracy (Percentage of people) and the
\end{abstract}


independent variable $\mathrm{X}=$ Income Inequality (Gini Coefficient) with an ANOVA significance of $0.00 \%$, an $\mathrm{R}^{\wedge} 2=86.3 \%$, of which indicates that the model has a good fit to the explanatory variable, with a sensitivity of $\beta=$ 0.438 .

Key words: Digital illiteracy, Income inequality, ICTs, Digital divide, Labor skills.

\section{Introducción:}

La globalización en el contexto del desarrollo social de la información y el conocimiento ha permitido que la tecnología y el desarrollo económico fluyan en forma vertiginosa en las últimas décadas. Esta evolución ha permitido la innovación creativa en diferentes ámbitos del sector laboral a nivel mundial (Acosta, Yagual, \& Coronel, 2018). Así mismo, se describe una frontera que marca el fin de la era industrial y el inicio de la era de la información. Este cambio ha generado nuevos procesos basados en perspectivas estratégicas como la financiera, cliente, procesos internos y aprendizaje, así mismo la creación de nuevas plazas de trabajo traen consigo mano de obra preparada para cumplir con los objetivos desde el punto de vista estratégico empresarial. Sin embargo, tiene incidencia en el sector público y privado (Kaplan \& Norton, 1992).

Por tanto, es relevante identificar los factores claves de un ecosistema digital donde las organizaciones dinamizan de forma competitiva aprovechando los adelantos tecnológicos dentro de una red de naturaleza abierta. Tal es el avance que la disrupción tecnológica ha desencadenado en entornos macrosociales como nuevos tipos de empresas, ciudades inteligentes, nuevos empleos, inclusión financiera, entre otros beneficios de la economía digital que involucran todos los sectores de la sociedad (Moreno, González, Torres, \& Araya, 2017).

En cuanto a la transformación e importantes cambios de las fuerzas productivas también existen cambios en el conjunto de los factores de producción, abarcando una incidencia en el comercio, consumo y crédito provocando el nacimiento de una nueva división del trabajo a niveles globales. Se inicia un nuevo ciclo industrial, encabezado por el sector electrónico - informático, que empezó a propiciar una lógica diferente de acumulación del capital.

Bajo este contexto, es imprescindible la generación de brechas digitales que manejen y cubran las necesidades de fuerza laboral cualificada para que el actual sistema económico funcione de forma eficiente (Guzmán, Muñoz, Álvarez, \& Velázquez, 2014). Por tanto, para que un objetivo estratégico se cristalice es necesario contar con participación del sistema de educación, el gobierno y la empresa de forma conjunta para actuar sobre las 
brechas desarrollando entornos de aprendizaje los cuales socialicen contenidos que garanticen la accesibilidad a la tecnología como equipos de cómputo, internet, software y demás herramientas tecnológicas bajo una perspectiva social, económica e incluyente que cubra a todos los participantes de la comunidad.

Tomando como partida las características fundamentales que el acortamiento de la brecha digital es imperante considerar que la alfabetización, ya que esta abarca no solo la capacidad de leer y escribir, también permite ser competitivos por tal razón es una competencia demostrada en el ámbito de las destrezas comunicativas. Esto permite el desarrollo del individuo con características funcionales, de acuerdo con la edad de forma independiente, en la sociedad dinamizando dentro de ella desde un punto de vista laboral. Aprender a interpretar signos y símbolos, es conseguir una forma de conectarse con la realidad mejorando las relaciones sociales en función de cambios y la dinámica social (Bawden, 2002).

Dentro del marco general de la alfabetización, la digitalización es un factor relevante, World Economic Forum (2012) describe esta conexión de acuerdo al coeficiente de correlación, el índice presenta un aumento del 10\% en digitalización representando un incremento de $6,4 \%$ en la dinámica innovadora, tanto dicha aceleración de la innovación es provocada por la introducción de servicios y aplicaciones proporcionados por las TICs, que adicionan nuevas aplicaciones y servicios (telemedicina, búsqueda en Internet, comercio electrónico, educación a distancia, redes sociales, etc.), como también nuevas formas de comercio e intermediación financiera (Jordán, Galperin, \& Pérez, 2013). En consecuencia, determinadas estimaciones, muestran que el aporte económico de la digitalización es significativo (ver tabla1).

Tabla 1.

América Latina: estimación del impacto económico de la digitalización

\begin{tabular}{|c|c|c|c|c|c|c|}
\hline \multirow[b]{2}{*}{ Pais } & \multicolumn{3}{|c|}{ Indicadores en 2011} & \multicolumn{3}{|c|}{$\begin{array}{l}\text { Cambio como resultado de un aumento de } 10 \% \\
\text { en el indice de digitalización }{ }^{\mathrm{a}}\end{array}$} \\
\hline & $\begin{array}{r}\text { Índice de } \\
\text { digitalización }\end{array}$ & $\begin{array}{r}\text { PIB per cápitab } \\
\text { (dólares) }\end{array}$ & $\begin{array}{r}\text { Índice de } \\
\text { innovación }\end{array}$ & $\begin{array}{r}\text { Índice de } \\
\text { digitalización }\end{array}$ & $\begin{array}{r}\text { PIB per } \\
\text { cápitab } \\
\text { (dólares) }\end{array}$ & $\begin{array}{r}\text { Índice de } \\
\text { innovación }\end{array}$ \\
\hline Argentina & 41,32 & 10881 & 34,40 & 45,45 & 10969 & 36,60 \\
\hline Brasil & 36,61 & 12594 & 36,60 & 40,27 & 12696 & 38,94 \\
\hline Chile & 45,33 & 13738 & 42,70 & 49,86 & 13849 & 45,43 \\
\hline Colombia & 38,33 & 7121 & 35,50 & 42,16 & 7179 & 37,77 \\
\hline Costa Rica & 37,33 & 8644 & 36,30 & 41,06 & 8714 & 38,62 \\
\hline Ecuador & 32,75 & 4504 & 28,50 & 36,03 & 4540 & 30,32 \\
\hline El Salvador & 29,56 & 3602 & 29,50 & 32,52 & 3631 & 31,39 \\
\hline México & 37,05 & 9980 & 32,90 & 40,76 & 10061 & 35,01 \\
\hline Panamá & 44,29 & 8740 & 30,90 & 48,72 & 8811 & 32,88 \\
\hline Paraguay & 28,68 & 3594 & 31,60 & 31,55 & 3623 & 33,62 \\
\hline Perú & 32,20 & 5860 & 34,10 & 35,42 & 5907 & 36,28 \\
\hline Uruguay & 42,78 & 14294 & 35,10 & 47,06 & 14410 & 37,35 \\
\hline
\end{tabular}


Nota: Esta tabla muestra información de (World Economic Forum, 2012); (Katz \& Koutroumpis, International Telecommunication Union, 2012)

Según el Banco Mundial, Ecuador en comparación con otros países de América Latina y el mundo, presenta una baja conectividad, el $43 \%$ de la población está conectada a internet (ver tabla 2). Sin embargo, World Economic Forum analiza la relación a las transformaciones 2.0 en congruencia con el progreso de Ecuador en el uso de las tecnologías de la información, bajando 8 posiciones del puesto 108 al 116, de entre 1398 países (Renó, Gosciola, \& Renó, 2018).

Tabla 2.

Conectividad por pais

\begin{tabular}{ll}
\hline Conectividad por país & Porcentaje \\
\hline Colombia & 52,6 \\
Chile & 72,4 \\
Bélgica & 85 \\
Bolivia & 39 \\
Canadá & 87 \\
Dinamarca & 96 \\
Ecuador & $\mathbf{4 3}$ \\
España & 76,2 \\
Estados Unidos & 87 \\
Marruecos & 56,8 \\
México & 44,4 \\
Senegal & 17,7
\end{tabular}

Nota: La tabla toma información de World Economic Forum; (Renó, Gosciola, \& Renó, 2018)

En Ecuador a pesar de que puede existir implementación de nuevas tecnologías en entornos laborables públicos como privados la brecha a cubrir es alta. El Instituto Nacional de Estadística y Censos (INEC) (2017), describe una relación de principales estadísticas para definir varias brechas que son necesarias cubrir y garantizar la incursión en la Economía Digital. Sin embargo, el INEC ofrece información relevante para analizar la evolución y situación actual de la incidencia tecnológica en los distintos ámbitos socioeconómicos.

La presencia de laptops o computadores portátiles en hogares ecuatorianos durante cinco años aumentó en 13,7 puntos, mientras que las computadoras de escritorio se han mantenido estable con una variación de 0,3 puntos (Ver figura1). 
Figura 1.

Equipamiento tecnológico del hogar a nivel nacional

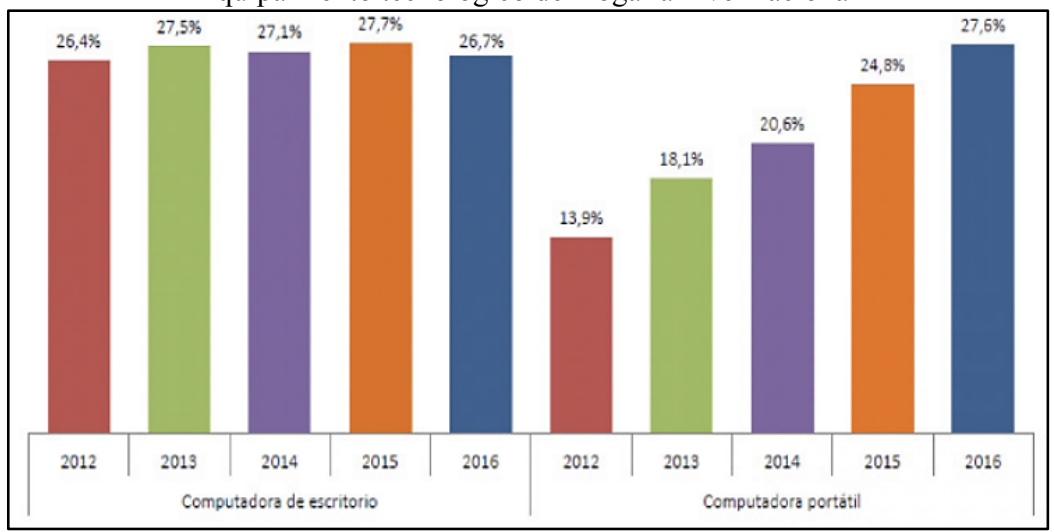

Nota. Información de la Encuesta Nacional de Empleo Desempleo y Subempleo - ENEMDU (2012 - 2016)

Este reporte también señala que el 36,0\% de los hogares cuentan con servicio de internet, donde el área urbana tiene mayor participación con un $44,6 \%$ en contraste con el área rural que cuenta con una participación menor al crecimiento de unos 11,6 puntos (Ver figura2).

Figura 2.

Acceso a internet según área

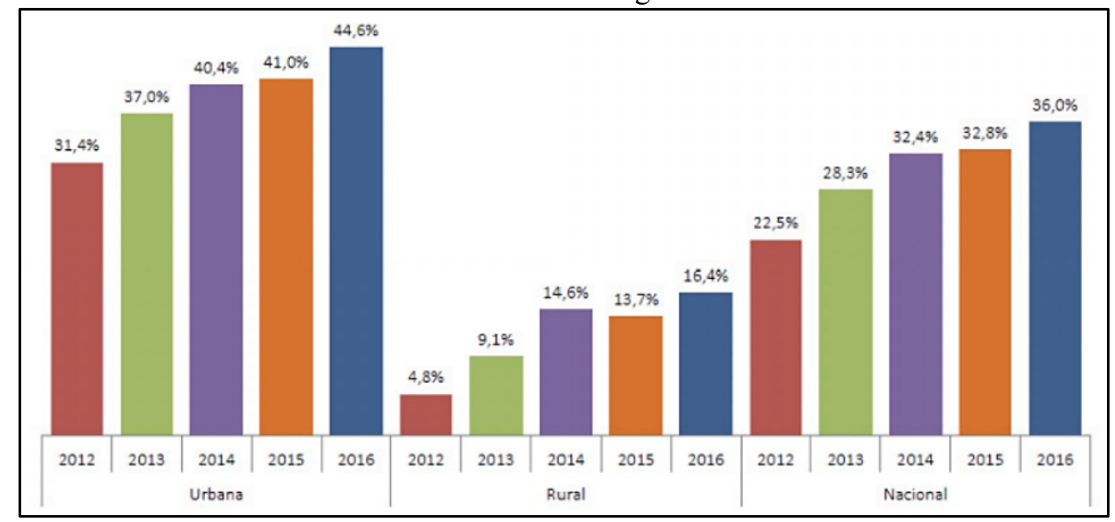

Nota: Información tomada de la Encuesta Nacional de Empleo Desempleo y Subempleo - ENEMDU $(2012-2016)$

En cuanto al analfabetismo digital el INEC determinó una tendencia decreciente a nivel nacional con una participación de $11,5 \%$, urbano $6,9 \%$ y a nivel rural del $22 \%$ lo que fundamenta la importancia de un accionar para este tipo de problema que abarca sectores sociales como económicos a nivel nacional (Ver figura3). 
Figura 3.

Porcentaje de personas analfacbetas digitales por área

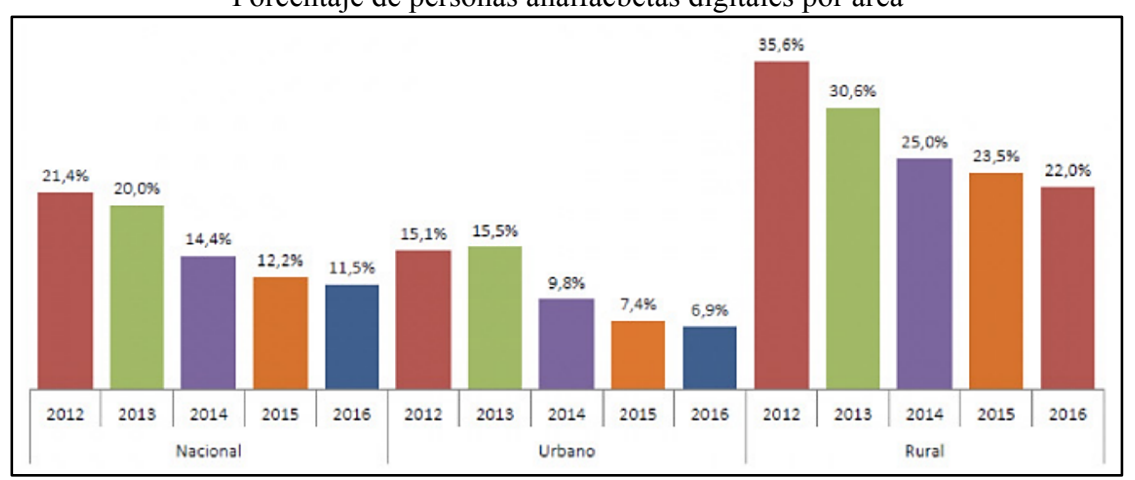

Nota: Información tomada de la Encuesta Nacional de Empleo Desempleo y Subempleo - ENEMDU $(2012-2016)$

Las consecuencias del analfabetismo digital desencadena un escaso conocimiento sobre la población nacional en las Tecnologías de Información y Comunicación provocando factores como la exclusión laboral, nivel social y económico preocupante que mantiene latente el subdesarrollo. Proyectos gubernamentales como la instalación de Infocentros han tenido un efecto positivo como la reducción del analfabetismo y brecha digital (Guallo \& Guadalupe, 2018).

La capacitación y práctica en constante evolución en entornos virtuales como el uso de aplicaciones informáticas en los dispositivos electrónicos donde el acceso a internet es imperante, elevan la probabilidad de mejorar la calidad de vida en las personas mediante la inclusión social y laboral. Sin embargo, dicho éxito tambien influyen en las actividades comerciales, económicas, servicios básicos, como el auge del uso de los servicios en línea que las tecnologías informáticas ofrecen permitiendo obtener calidad de servicio y tiempos de respuestas eficientes en las transacciones.

\section{Metodología}

El estudio metodológico del presente artículo definirá que tipo de análisis se ejecutará. De acuerdo con Katz y Koutroumpis (2012) donde describe que más allá del impacto del acceso a banda ancha, este estudio se basa en la relación combinada del conjunto de servicios y aplicaciones posibilitados por la banda ancha; siguiendo el argumento de asequibilidad planteado por Galperin y Ruzzier (2010) determina al tercer factor explicativo de la brecha de demanda digital, que es el económico. Para ello, el desarrollo del concepto de digitalización que define la capacidad de utilizar tecnologías digitales para generar, procesar y compartir información está relacionado con el enfoque de penetración de la tecnología como el uso 
de aplicaciones y el consumo de contenidos en tres niveles: i) individuos, empresas y gobierno, ii) procesos de producción de bienes y servicios y iii) provisión de servicios públicos.

Por lo tanto, mediante un enfoque cuantitativo del cual se pretende determinar un alcance descriptivo, inferencial y correlacional basado en un diseño no experimental transeccional en los periodos desde el 2008 hasta el 2019 cuyas variables a relacionar son la tasa nacional de personas analfabetas digitales y la desigualdad por ingresos por medio del coeficiente de Gini, este método estadístico no paramétrico se aplica para detectar relaciones causales donde los datos utilizados se los evalúa a través de la regresión lineal simple.

La investigación propuesta relaciona el método de investigación empírica y la epistemología correspondiente propuesta por Figueroa (2012, p. 109) donde aparte de la fundamentación teórica también posee base de datos que permitan la validación de variables. La epistemología aplicada es la Popperiana, con su respectivo análisis cuantitativo alineado con el objetivo de investigación explicativo. La aplicación de la prueba estadística $\beta$ como prueba empírica.

\section{Identificación de las variables}

$\mathrm{X}$ : Desigualdad Ingresos (Coeficiente Gini)

$\mathrm{Y}: \quad$ Analfabetismo digital (Porcentaje personas)

Tabla 3.

Matriz de variables

\begin{tabular}{lcc}
\hline \multicolumn{1}{c}{ Descripción } & Tipo de variable & Causalidad \\
\hline Analfabetismo digital & Endógena & \\
Desigualdad por ingresos & Exógena & $(-)$ \\
\hline
\end{tabular}

\section{Técnica estadística}

\section{Población}

La población de estudio considerada por la encuesta tecnológica por el INEC (2020) y la Encuesta Nacional de Empleo, Desempleo y Subempleo (ENEMDU, 2019), los periodos a considerar desde el 2008 hasta el 2019. Donde los datos fueron ajustados en $\mathrm{N}=12$. 
Tabla 4.

Variables

\begin{tabular}{ccc}
\hline Año & $\begin{array}{c}\text { Porcentaje de personas } \\
\text { analfabetas digitales }\end{array}$ & $\begin{array}{c}\text { Desigualdad de ingresos } \\
\text { coeficiente de Gini }\end{array}$ \\
\hline 2008 & 32,42 & 0,551 \\
2009 & 33,60 & 0,515 \\
2010 & 29,22 & 0,504 \\
2011 & 25,14 & 0,505 \\
2012 & 21,38 & 0,473 \\
2013 & 20,04 & 0,477 \\
2014 & 14,43 & 0,485 \\
2015 & 12,22 & 0,467 \\
2016 & 11,45 & 0,476 \\
2017 & 10,48 & 0,466 \\
2018 & 10,68 & 0,459 \\
2019 & 11,39 & 0,478 \\
\hline
\end{tabular}

Fuente: (INEC, 2020); (ENEMDU, 2019)

\section{Muestra}

En función de la limitación de la información se consideró trabajar con toda la población, por lo tanto, no se aplicó ningún tipo de muestreo. Se procedió a tomar todos los datos como población.

\section{Prueba estadística utilizada}

Como en este caso de estudio se determinaron una variable exógena y una endógena, por lo que la metodología propuesta denota la aplicación de la regresión lineal simple, estableciendo una prueba de hipótesis que permita someter la misma al proceso de falsación.

\section{Supuestos considerados}

a. Con respecto al modelo de regresión lineal simple:

$$
\begin{aligned}
& Y=F(X) \\
& Y=\beta_{0}+\beta_{1} X
\end{aligned}
$$

b. Con respecto a las varianzas: varianzas iguales (condición de homocedasticidad)

\section{Hipótesis propuesta}

\section{Hipótesis Nula}

$H_{0}: \beta=0$, "Un aumento en el analfabetismo digital no incide en la desigualdad por ingresos"

Hipótesis Alternativa

$H_{1}: \beta \neq 0$, "Un aumento en el analfabetismo digital, incide en la desigualdad por ingresos" 


\section{Criterios de decisión}

Si el p-value < 0.05; rechazar Ho, si el p-value $>0.05$; aceptar Ho

\section{RESULTADOS}

Mediante el uso del software IBM SPSS Statistics 22, se procedió a realizar el modelo de Regresión Lineal Simple del cual se obtuvo una sensibilidad de $\beta=0.438$.

En primera instancia se realizó el análisis descriptivo de las dos variables propuestas en la presente investigación.

Tabla 5.

Estadísticos descriptivos

\begin{tabular}{|c|c|c|c|}
\hline Variables & Media & $\begin{array}{l}\text { Desviación } \\
\text { estándar }\end{array}$ & $\mathbf{N}$ \\
\hline $\begin{array}{c}\text { Analfabetismo digital } \\
\text { (Porcentaje de personas) }\end{array}$ & 19.37 & 8.86 & 12 \\
\hline $\begin{array}{l}\text { Desigualdad en los Ingresos } \\
\text { (Coeficiente de Gini) }\end{array}$ & 0.48 & 0.026 & 12 \\
\hline
\end{tabular}

De acuerdo a la Tabla 5 se observa que, de los 12 años analizados, la media del porcentaje del Analfabetismo digital es 19.37 y una desviación de 8.86 y la media del coeficiente de Gini en la Desigualdad de ingresos es de 0.48 y la desviación de 0.026 .

Tabla 6.

Coeficientes Modelo de Regresión Lineal Simple

\begin{tabular}{llccccc}
\hline \multicolumn{1}{c}{ Modelo } & \multicolumn{2}{c}{$\begin{array}{c}\text { Coeficientes no } \\
\text { estandarizados }\end{array}$} & $\begin{array}{c}\text { Coeficientes } \\
\text { tipificados } \\
\text { Beta }\end{array}$ & T & Sig. \\
& B & Error típ. & Beta & & \\
\hline (Constante) & &, 438 &, 010 & & 43,687 &, 000 \\
$\begin{array}{l}\text { Desigualdad en los } \\
\text { Ingresos (Coeficiente }\end{array}$ &, 003 &, 000 &, 863 & 5,394 &, 000 \\
Gini) & & & & & \\
\hline
\end{tabular}

Nota: Variable dependiente: Analfabetismo digital

De acuerdo con la Tabla 6 se observa que la constante del modelo es $\beta=0.438$, la variable independiente tiene un $\mathrm{p}$-value $<0.05$, lo cual quiere decir que esta variable explica a la variable dependiente. 
Figura 4.

Relación entre analfabetismo digital y los ingresos

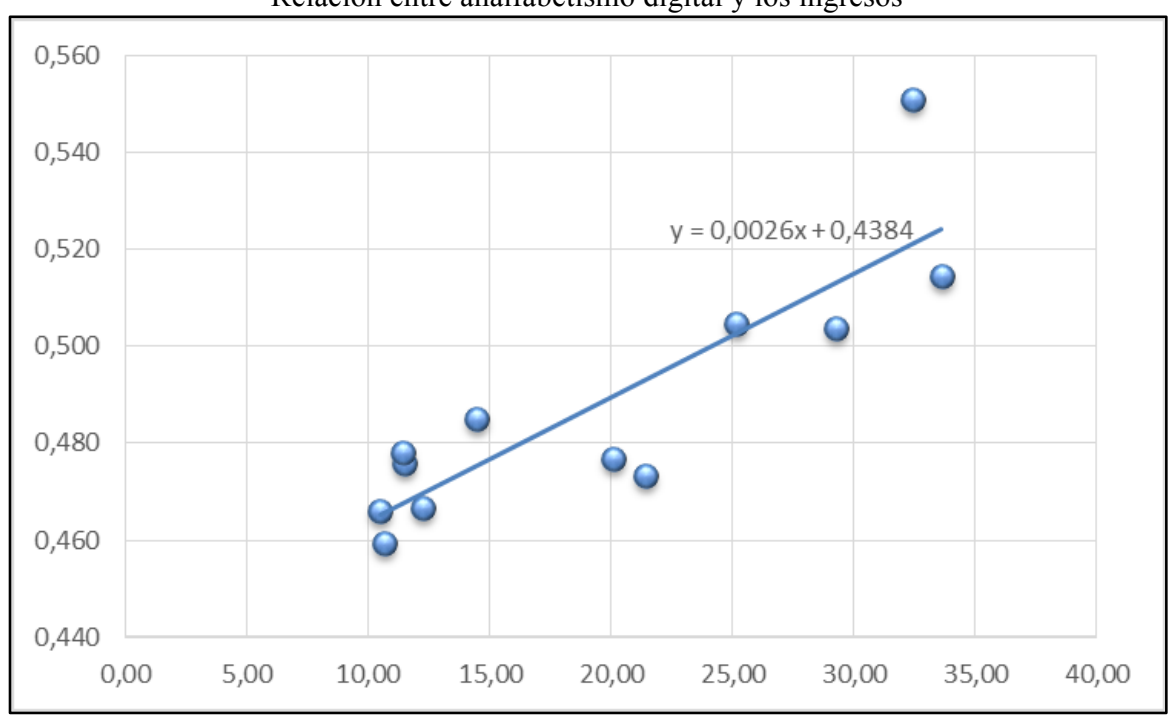

Nota: Resultados utilizando SPSS - IBM

Como se puede observar en la Figura 4 los puntos de dispersión se encuentran cerca a la recta, por lo tanto se demuestra que los datos no estan muy dispersos con repecto a su media.

\section{Ecuación del Modelo de Regresión Lineal Simple}

$$
Y=0.438+0.03 x
$$

De acuerdo con el modelo propuesto se interpreta de la siguiente manera:

Por un aumento en el analfabetismo digital (Porcentaje de personas) incrementaría en 0.03 la desigualdad en los ingresos.

El p-value de la regresión lineal simple es igual a 0.00 , error, tomando en consideración un nivel de significancia fijado (5\%); por lo tanto, se rechaza la hipótesis nula de $\beta=0$ y se acepta la hipótesis alternativa que $\beta \neq 0$, lo que implica que existe relación estadística. 


\section{Correlación de las Variables}

Tabla 7.

Correlación

\begin{tabular}{ccc}
\hline Variables & $\begin{array}{c}\text { Analfabetismo digital } \\
\text { (Porcentaje de } \\
\text { personas) }\end{array}$ & $\begin{array}{c}\text { Desigualdad en los } \\
\text { Ingresos (coeficiente } \\
\text { Gini) }\end{array}$ \\
\hline $\begin{array}{c}\text { Analfabetismo digital (Porcentaje de personas) } \\
\text { Desigualdad en los Ingresos (coeficiente Gini) }\end{array}$ & 1 & $0.863^{* *}$ \\
\hline
\end{tabular}

Nota: **. La correlación es significativa en el nivel 0,01 (2 colas).

Se observa que el coeficiente de correlación es 0.863 , con una significancia de 0.01. De acuerdo al análisis de los valores de Pearson van de -1 a 1 , siendo 0 el indicador de que no existe correlación, se determina que entre estas dos variables existe una correlación alta ya que no es cero, sino que se inclina al 1. La dirección de la correlación es positiva, es decir es directa, por lo tanto al aumentar el Analfabetismo digital, aumentaría la desigualdad en los Ingresos.

A continuación, se muestra el ajuste del modelo utilizando como criterio el porcentaje de variación $R^{2}$ que es igual a $86.3 \%$, que está cerca al 1, esto quiere decir que es alto, por ende, la variable dependiente es explicada por la variable independiente.

Tabla 8.

Resumen del modelo

\begin{tabular}{cccccc}
\hline Modelo & $\mathbf{R}$ & R cuadrado & $\begin{array}{c}\text { R cuadrado } \\
\text { corregida }\end{array}$ & $\begin{array}{c}\text { Error típ. de la } \\
\text { estimación }\end{array}$ & $\begin{array}{c}\text { Durbin- } \\
\text { Watson }\end{array}$ \\
\hline 1 &, $863^{\text {a }}$ &, 744 &, 719 &, 01395 & 1,742 \\
\hline
\end{tabular}

Nota: a. Variables predictoras: (Desigualdad Ingresos), b. Variable dependiente: Analfabetismo digital Luego se procedió a analizar el nivel de significancia por medio de ANOVA, y observar si existe relación entre la variable dependiente $Y=$ Analfabetismo digital (Porcentaje personas) y la variable independiente $\mathrm{X}=$ Ingresos

(Coeficiente GINI).

Tabla 9.

ANOVA

\begin{tabular}{lccccc}
\hline Modelo & $\begin{array}{c}\text { Suma de } \\
\text { cuadrados }\end{array}$ & Gl & $\begin{array}{c}\text { Media } \\
\text { cuadrática }\end{array}$ & F & Sig. \\
\hline Regresión &, 006 & 1 &, 006 & 29,100 &, $000^{\mathrm{b}}$ \\
Residual &, 002 & 10 &, 000 & & \\
Total &, 008 & 11 & & & \\
\hline
\end{tabular}

Nota: a. Variable dependiente: Analfabetismo digital (Porcentaje personas) 
Como se puede observar en la Tabla 9 la significancia de la ANOVA es $0.00 \%$, esto significa que las dos variables están relacionadas y por tanto hay diferencias significativa entre los grupos.

\section{Conclusión}

Las barreras que existen en la formación digital a partir del analfabetismo digital determinan la desigualdad de posibilidades de acceso a la información y al conocimiento, sin embargo, no es sólo una perspectiva tecnológica, debido a que también son necesarios considerar factores socioeconómicos tales como: la capacidad de adquirir equipos de cómputo, infraestructuras tecnológicas y la formación. Así mismo, se confirma desde un punto de vista teórico-conceptual que la brecha digital no genera efectos directos o unidireccional en el desarrollo de riqueza, oportunidades y bienestar, sino que, además incide de forma negativa en la disminución de pobreza y la exclusión social. En consecuencia, se podría considerar como un alto riesgo para la sociedad de la información y paralelamente en el distanciamiento de la brecha entre pobres y ricos.

Estos efectos a nivel de equipamiento e infraestructura tecnológica en Ecuador en relación con la formación digital, se debe en gran mediad, como efecto de desigualdades socioeconómicas, debido a la incidencia en grupos desfavorecidos, transformándose en desventaja para el desarrollo de una transformación tecnológica, así mismo, disminuye las oportunidades sociales y económicas. Esto quiere decir que, a nivel regional si se valora la brecha digital entre países, se observa que la brecha digital incide en el desarrollo o el retraso económico de un país.

Como recomendación para nuevas investigaciones, se debe analizar también la exclusión social enlazada a las nuevas tecnologías. Debido a que conlleva a nuevos escenarios complejos en materia de desintegración social, además del acceso a las nuevas tecnologías o el no acceso a la información, identificada como una exclusión o marginación institucional, como efectos podrían llegar a no acceder a nuevas formas de consumo y comunicación o a servicios públicos básicos.

\section{Referencias:}

Lazový, J., \& Sipko, J. (2014). Impact of Financial Derivatives on the Real Economy. International Journal of Management Excellence , 4 (1).

(n.d.). From www.sbp.org.pk

(n.d.). From www.secp.org.pk

(n.d.). From www.kse.pk 
Zeithaml, V. (1988). "Consumer perceptions of price, quality and value: a means-end model and synthesis of evidence". Journal of Marketing , 52 (3), 2-22.

Schiffman, L. G., \& Lazar Kanuk, L. (2010). Comportamiento del Consumidor (DÉCIMA EDICIÓN ed.). México: PEARSON EDUCACIÓN.

Washburn, J. H., \& Plank, R. E. (2002). "Measuring Brand Equity: An evaluation of a consumer-based brand equity scale". Journal of Marketing Theory and Practice , 10 (1), 4662 .

Wood, L. (2000). "Brands and brand equity: definition and management". Management Decision , 38 (9), 662-669.

World Economic Forum. (2012). The Global Information Technology Report 212. Ginebra: INSEAD-WEf.

WTTC. (2011). Travel and Tourism Eonomic Impact. WTTC,London.

Yoo, B., \& Donthu, N. (2001). "Developing and validating a multidimensional consumerbased brand equity scale". Journal of Business Research, 52, 1-14.

Yoo, B., Donthu, N., \& Lee, S. (2000). "An examination of selected marketing mix elements and brand equity". Journal of the Academy of Marketing Science , 28 (2), 195-211.

Vaz, C. R., Shoeninger Rauen, T. R., \& Rojas Lezana, Á. G. (2017). Sustainability and Innovation in the Automotive Sector: A Structured Content Analysis. MDPI .

Villarejo-Ramos, A. F., \& Sanchez-Franco, M. J. (2005). "The impact of marketing communication and price promotion on brand equity". Journal of Brand Management , 12 (6), 431-444.

Aaker, A. (1996). "Measuring brand equity across products and markets". California Management Review, 38 (3), 102-120.

Aaker, A. (1991). Managing Brand Equity: Capitalizing on the value of brand name. New York: The Free Press.

Acosta, M., Yagual, A., \& Coronel, V. (2018). Perspectivas de la economía digital en Latinoamerica: Caso Ecuador. 3C Empresa: Investigación y pensamiento crítico, 28-43.

Afaqi, M. J., \& Seth, N. J. (2007). SME Sector, Genesis, Challanges \& Prospects. Islamabad: SMEDA.

Ahmed, V., \& Batool, S. India-Pakistan Trade: Perspective from the Automobile Sector in Pakistan. In N. Taneja, I. Dayal, N. Taneja, \& I. Daniyal (Eds.), India-Pakistan Trade Normalization, The Unfinished Economic Agenda. New Delhi, India: Springer Nature.

Alí, A.-B., Francisco, V.-M., \& Gilberto, P.-L. (2016). Impact of derivatives markets on economic growth in some of the major world economies A difference-GMM panel data estimation (2002-2014). THE IEB INTERNATIONAL JOURNAL OF FINANCE , 110-127.

Analysis, $\quad \mathrm{S}$

http://spearheadresearch.org/index.php/category/researchopinions/ecofin.

(2016).

spearheadresearch.org: http://www.spearheadresearch.org

Analysis, S.

From

http://spearheadresearch.org/index.php/category/researchopinions/ecofin.

(2016)

spearheadresearch.org: http://www.spearheadresearch.org

Aqil, M., Qadeer, S., Ahmed, D. R., \& Qureshi, M. A. (2014). PROTECTION AND INDIGENIZATION LEVELS IN PAKISTAN AUTOMOBILE INDUSTRY FROM 1995 TO 2005. International Journal of Engineering \& Scientific Research , Vol.2 (Issue 4), 1-12. Atilgan, E., Aksoy, S., \& Akinci, S. (2005). "Determinants of brand equity: A verification approach in the baverage industry in Turkey". Marketing Intelligence and Planning , 23 (3), 237-248.

Buil, I., Martinez, E., \& de Chernatony, L. (2013). "The influence of brand equity on consumer responses". Journal of Consumer Marketing , 30 (1), 62-74. 
Bawden, D. (2002). Revisión de los conceptos de alfabetización informacional y alfabetización digital. Revistas Científicas de la Universidad de Murcia , 361-408.

Bank, T. A. (2008). Private Sector Assessment Pakistan. Pakistan: The Asian Developement Bank.

Bashir, R., Shakir, R., Ashfaq, B., \& Hassan, A. (2014). The Efficiency of Foreign Exchange Markets in Pakistan: An Empirical Analysis. The Lahore Journal of Economics , 19 (1), 133-149.

Barwise, P. (1993). "Brand equity: Snark or Boojum?". International Journal of Research in Marketing , 10 (1), 93-104.

Bekaerta, G., \& Harvey, C. R. (n.d.). Research in emerging markets finance: looking to the future. COLUMBIA BUSINESS SCHOOL.

Bekale, A. N., Botha, E., \& Vermeulen, J. (March de 2015). Institutionalisation of Derivatives Trading and Economic Growth: Evidence from South Africa. ERSA working paper 505 . Economic Research Southern Africa (ERSA).

Berthon, P. R., Pitt, L. F., \& Cambell, C. (2008). "Ad lib: when customers create the ad". CA Management Review , 50 (4), 6-31.

BKPM. (2017). 4th Quarterly Investment Report Final. Ghana Investment Promotion Center.

Blackwell, R. D., Miniard, P. W., \& Engel, J. F. (2002). Comportamiento del Consumidor. Mexico: International Thomson Editores.

Bruhn, M., Schoenmueller, V., \& Schäfer, D. (2012). "Are social media replacing traditional media in terms of brand equity creation?". Management Research Review , 35 (9), 770-790.

CWIG. (2004). Stats Ghana report. 1-99: http://www.statsghana.gov.gh/docfilescwiq report. Calistru, R. A. (2012). The credit derivatives market a threat to financial stability? 8th International Strategic Management Conference (pp. 552 - 559). ELSEVIER.

Calistru, R. A., \& Costuleanu, C. (2011). THE IMPACT OF DERIVATIVES ON MARKET FUNCTIONING. ANNALS OF THE UNIVERSITY OF CRAIOVA ECONOMIC SCIENCES . Carmines, E. G., \& McIver, J. P. (1981). "Analyzing models with unobserved variables". In Steffel R.V.,\& Ellis,R.S.(Eds). Structural and social bond of commitment in inter-firm relationships. Journal of Applied Business and Ecomomics , 10 (1), 1-18.

Chaudhuri, A. (1995). "Brand equity or double jeopardy?". Journal of Product and Brand Management , 7 (2), 26-32.

Chaudhuri, A. (1999). "Does brand loyalty mediate brand equity outcomes?". Journal of Marketing Theory and Practice, 7 (2), 136-146.

Chaudhuri, A., \& Holbrook, M. (2001). "The chain of effects from brand trust and brand affect to brand performance: the role of brand loyalty". Journal of Marketing , 65, 81-93.

Chen, Y., Lin Lawell, C.-Y. C., \& Wang, Y. (2017). The Chinese Automobile Industry and Government Policy.

Chen, A. C.-H. (2001). "Using free associations to examine the relationship between the characteristics of brand associations and brand equity". Journal of Product and Brand Management, 10 (7), 439-451.

Chengedzai , M., Manillall , D., \& Lawrence, M. (2 de Abril de 2014). 'Shopper typologies amongst a Generation $Y$ consumercohort and variations in terms of age in the fashionapparel market'. Retrieved 26 de Enero de 2015 from http://www.actacommercii.co.za: http://dx.doi.org/10.4102/ac.v14i1.209

Cobb-Walgren, C. J., Ruble, C. A., \& Donthu, N. (1995). "Brand equity, brand preference and purchase intend". Journal of Advertising , 24 (3), 25-41.

Craig, C., \& Douglas, S. (2000). International Marketing Research (2nd ed.). Chichester: John Wiley \& Sons. 
Evans, Y. (2018). Foreign Direct Investment In Ghana:The Distribution Among the Sectors and Regions. International Journal of Current Research .

(2012-13). Economic Survey of Pakistan.

ENEMDU. (2019). Encuesta Nacional de Empleo, Desempleo y Subempleo (ENEMDU). Boletín Técnico - $N^{\circ}$ 02-2019-ENEMDU .

Erdem, T., Swait, J., \& Louviere, J. (2002). "The impact of brand credibility on consumer price sensibility". Internartional Journal of research in marketing , 19, 1-19.

D.Tang, K. (2012). China-Africa Foreign Trade Policies: The Impact of China's Foreign Direct Investment(FDI) Flow on Employment of Ghana. Energy Procedia , 553-557.

del Rio, A. B., Vazquez, R., \& Iglesias, V. (2001). " The effects of brand associations on consumer response". Journal of Consumer Marketing , 18 (5), 410-425.

Doyle, P. (2001). "Shareholder-value-based brand strategies". Journal of Brand Management, 9 (1), 20-30.

Dodd, R. (October de 2002). Derivatives, the Shape of International Capital Flows and the Virtues of Prudential Regulation. Discussion Paper No. 2002/93 . World Institure of Developement Economic Research (WIDER).

Fund, C. S. (2006). Policy Analysis on the Competitive Advantage of the Motorcycle Industry in Pakistan; Problems and Prospects. Islamabad: USAID \& Ministry of Finance Govt of Pakistan.

(2006). Feasibility Introducing Exchange traded Derivatives in Pakistan .

Figueroa, A. (2012). The Alpha-Beta Method. Scientific rules for economics and the social sicences.

Foux, G. (2006). "Consumer-generated media: get your customers involved". Brand Strategy, 38-39.

Fournier, S., \& Avery, J. (2011). "The uninvited brand". Business Horizons , 54 (3), $193-$ 207.

Ford, C. V. (1996). Lies! Lies!! Lies!!! The Psychology of Deceit. Washington, DC: American Psychiatric Press, Inc.

Guzmán, J., Muñoz, J., Álvarez, F., \& Velázquez, C. (2014). La brecha digital en el estado de Aguascalientes. Investigación y Ciencia, 54-61.

Guallo, J., \& Guadalupe, S. (2018). La alfabetización digital en Ecuador en el siglo XXI. Revista Atlante: Cuadernos de Educación y Desarrollo .

Gürkaynak, R. S., \& Wolfers, J. (December de 2005). Macroeconomic Derivatives An Initial Analysis of Market-Based Macro Forecasts, Uncertainty and Risk. IZA DP No. 1899 . Gautam, I., \& Kavidayal, P. (2016). EVOLUTION AND FUTURE PROSPECTS OF INDIAN DERIVATIVE MARKET. International Journal in Management and Social Science, 4 (3).

Gallager, K., Foster, K. D., \& Parson, J. (2001). "The medium is not the message: advertising effectiveness and content evaluation in print and on the web". Journal of Advertising Research , 41 (4), 57-70.

Galperin, H., \& Ruzzier, C. (2010). Las tarifas de banda ancha: benchmarking y análisis en $V$. Jordán, H. Galperin y W. Peres, Acelerando la revolución digital: banda ancha para América Latina y el Caribe. Santiago de Chile: CEPAL.

Gensler, S., Völckner, F., Liu-Thompkins, Y., \& Wiertz, C. (2013). "Managing brands in the social media environment". Journal of Interactive Marketing , 27 (4), 242-256.

Godes, D., \& Mayzlin, D. (2009). "Firm-created word-of-mouth communication: evidence from a field test". Marketing Science, 28 (4), 721-739.

Grover, R., \& Srinivasan, V. (1992). "Evaluating the multiple effects of retail promotions on brand-loyalty and brand- switching segments". Journal of Marketing Research , 29, 76-89. 
Ilyas, H. (September de 2015). Research Gate. From https://www.researchgate.net/publication/281967253.

INEC. (2020). Encuesta tecnológicas. Tecnologías de la Información y Comunicación-TIC .

INEC. (2017). Instituto Nacional de Estadísticas y Censos. From http://www.ecuadorencifras.gob.ec/sociedad-de-la-informacion/

Iqbal, J. (2008). Stock Market in Pakistan - An Overview.

Hu, L. -T., \& Bentler, P. (1999). "Cutoff criteria for fit indexes in covariance srtucture analysis: Conventional criteria versus new alternatives". Structural Equation Modeling , 6, $1-55$.

Hussain, I. (September de 2012). PROSPECTS, CHALLENGES AND RISKS FOR INCREASING INDIA-PAKISTAN TRADE. IBA Working Paper Series , pp. 1-39.

Habibi, M. R., Laroche, M., \& Richard, M.-O. (2014). "Brand communities based in social media: How unique are they? Evidence from two exemplary brand communities". International Journal of Information Management, 34, 123-132.

Hair, J. F., Black, W. C., Babin, B. J., Anderson, R. E., \& Tatham, R. L. (2006). Multivariate data analysis (Vol. 6). New York: Pearson Prentice Hall, Upper Saddle River.

Hair, J. F., Jr, Anderson, R. E., Babin, B. E., \& Black, W. C. (2010). Multivariate data analysis (7th edition ed.). New Jersey: Prentice-Hall.

Hair, J. F., Ringle, C. M., \& Sarstedt, M. (2011). "PLS_SEM: Indeed a silver bullet". Journal of Marketing Theory and Practice , 19 (2), 139-151.

Hanna, R., Rohm, A., \& Crittenden, V. L. (2011). "We're all connected: The power of the social media ecosystem". Business Horizons , 54 (3), 265-273.

Jaffery, A. A., \& Mansoor, A. (2016). Competitive Analysis of Auto Sector in Pakistan and China.

Jianxi, L. a. (n.d.). The impact of government policies on industrial evolution : the case of China's automotive industry.

Johansson, J. K., \& Carlson, K. A. (2015). Contemporary Brand Management. Los Angelos: Sage.

Jordán, V., Galperin, H., \& Pérez, W. (2013). Banda ancha en América Latina: más allá de la conectividad. Santiago de Chile: Naciones Unidas.

Kusi, G. (2013). Regulatory Framework for Investing in Ghana. Ghana Investment Promotion Centre.

K.Boakye, F. F. (2013). Assessing Ghana's Contemporary Tourism Development Experience. Journal of Global Initiatives:Policy,Pedagogy,Perspective .

Kaplan, A. M., \& Haenlein, M. (2010). "Users of the world, unite! The challenges and opportunities of social media". Business Horizons , 53 (1), 59-68.

Kaplan, A. M., \& Haenlein, M. (2012). "The britney spears universe: social media and viral marketing at its best". Business Horizons , 55 (1), 27-31.

Kaplan, R., \& Norton, D. (1992). "The balanced scorecard- Measures that drive performance". Harvard Business Review .

Katz, R., \& Koutroumpis, P. (2012). Measuring Socio-Economic Digitization: A Paradigm Shift. unpublished manuscript .

Katz, R., \& Koutroumpis, P. (2012). The economic impact of broadband: case studies of the Philippines and Panama. Geneva.

Keller, K. (2009). "Building strong brands in a modern marketing communication". Journal of Marketing Communication, 12 (2-3), 139-155.

Keller, K. L. (2003). Strategic Brand Management: Building, Measuring and Managing Brand Equity (2nd ed.). New York: Prentice-Hall,Englewood Cliffs.

Keller, K. L., \& Lehmann, D. R. (2003). "How do brands create value?". Marketing Management, 12 (3), 26-31. 
Kim, Y.-K., \& Mugo-Waweru, F. (n.d.). Factors Behind Exchange-Traded Derivatives Products Success.

Kim, A. J., \& Ko, E. (2012). "Do social media marketing activities enhance customer equity? An empirical study of luxury fashion brand". Journal of Business Research , 65 (10), 1480-1486.

Kirmani, A., \& Wright, P. (1989). "Money talks: perceived advertising expense and expected product quality". Journal of Consumer Research , 16 (3), 344-353.

Kirtis, A. K., \& Karahan, F. (2011). "To be or not to be in social media arena as the most cost-efficient marketing stategy after the global recession". Procedia-Social and Behavioral Sciences , 24, 260-268.

Khalid, A. M. (2007). Bond Market Developments in Emerging Markets: Propsects and Challenges for Pakistan. SBP Research Bulletin, 3 (1) .

Khan, M. R., \& Ahmad, Y. (2012). FAILURE OF AUTOMOBILE MANUFACTURING IN PAKISTAN- A CASE OF THE REVO CAR. INTERDISCIPLINARY JOURNAL OF CONTEMPORARY RESEARCH IN BUSINESS , VOL 4 (No. 4), 1-16.

Khan, S. U., \& Abbas, Z. (2013). Does Equity Derivatives Trading Affect the Systematic Risk of the Underlying Stocks in an Emerging Market: Evidence from Pakistan's Futures Market. The Lahore Journal of Economics , 18 (1), 63-80.

Kotler, P. (2000). Marketing Management: Analysis, Planning, Implementation and Control (10 ed.). Englewood Cliff, NJ: Prentice Hall.

Kotler, P., \& Keller, K. L. (2012). A framework for marketing management (5th ed.). Boston: Prentice-Hall.

Lassar, W., Mittal, B., \& Sharma, A. (1995). "Measuring customer-based brand equity". Journal of Consumer Marketing , 12 (4), 11-20.

Lewis, M., \& Saarni, C. (Eds.). (1993). Lying and Deception in Everyday Life. New York: The Guilford Press.

Low, G., \& Lamb, C. J. (2000). "The measurement and dimensionality of brand associations". Journal of Product and Brand Management , 9 (6), 350-370.

Naz, F. (January de 2011). Pakistan Equity Derivative Market. Pakistan Business Review , 844-852.

Nag, B. (2016). Assessing the Future of Trade in the Automobile Sector between India and Pakistan: Implications of Abolishing the Negative List. New Delhi, India: Indian Institute of Foreign Trade New Delhi India.

Nag, B., Banerjee, S., \& Chatterjee, R. (2007). Changing Features of the Automobile Industry in Asia: Comparison of Production, Trade and Market Structure in Selected Countries. Asia-Pacific Research and Training Network on Trade Working Paper Series, No. 37, July $2007,1-47$.

Naqvi, N. (2013). Capital Market and Economic Growth. Business Recorder.

Netemeyer, R., Krishnan, B., Pulling, C., Wang, G., Yagci, M., Dean, D., et al. (2004). "Developing and validating measures of facets of consumer-based brand equity". Journal of Business Research , 57, 209-224.

Mukherjee, A., \& Sastry, T. (1996). The Automotive Industry in Emerging Economies:A Comparison of Korea, Brazil, China and India. Ahmedabad, India: Indian Institute of Management, Ahmedabad 380015, India.

M.Shafi, A. R., Hassan, S. S., \& Fareed, A. (2014). Impact of Derivatives on Efficiency of Stock Market: Evidence from Karachi Stock Exchange. International Journal of Scientific \& Engineering Research , 5 (7).

MacKay, M. (2001). "Evaluation of brand equity measures:further empirical evidence". Journal of product and brand management , 10 (1), 38-51. 
Mafini, C., Dhurup, M., \& Mandhlazi, L. (2014). Shopper typologies amongst a Generation $\mathrm{Y}$ consumer cohort and variations in terms of age in the fashion apparel market. Acta Commercii .

Mahfooz, Y. (2015). "Brand equity-consequence relationship: Evidence from automobile industry". International Journal of Business and Management , 10 (3), 81-90.

Malhotra, N. (2010). Marketing Research: An applied orientation (6th ed.). Prentice Hall.

Mangold, W. G., \& Faulds, D. J. (2009). "Social media :The new hybrid element of the promotion mix". Business Horizons , 52 (4), 357-365.

Martin, G. S., \& Brown, T. J. (1990). "In search of brand equity: the conceptualization and measurement of the brand impression construct". Marketing Theory and Applications , 2, 431-438.

McKee, S. (2010). Creative B2B Branding( No, Really): Building a Cretaive brand in a Business World. USA: Goodfellow Publisher Limited.

McLean, R. D., Pontiff, J., \& Zhao, M. (aUGUST de 2017). A Closer Look at the Effects of Equity Market Liberalization in Emerging Markets.

Mishra, B., Malik, S., \& Pore, L. (n.d.). Impact of Increased Derivatives-Trading in India on the Price-Discovery Process.

Mirza, M. S., \& Manarvi, I. A. (2011). Analysis of Technological advancements in Pakistani Automobile Car Industry. Global Journal of Research in Engineering , Volume 11 (Issue 3), $1-16$.

Modi, S. (2016). Material Qualification in the Automotive Industry. Centre for Automotive Research.

Moreno, N., González, A., Torres, A., \& Araya, J. (2017). Alfabetización digital a padres de familia en el uso de las redes sociales. Alteridad , 8-19.

Pakistan equity market. (December de 2012). From www.mugheescity.com

Pappu, R., Quester, P. G., \& Cooksey, R. W. (2005). "Consumer-based brand equity: improving the measurement-empirical evidence". Journal of Product and Brand Management , 14 (3), 143-154.

Pasha, H., \& Ismail, Z. (2012). An Overview of Trends in the Automotive Sector and the Policy Framework. International Growth Centre.

Parker, R. S., Hermans, C. M., \& Schaefer, A. D. (2004). "Fashion consciousness of Chinese, Japanese and American teenagers". Journal of Fashion Marketing and Management: An International Journal, 176-186.

Pike, S., Bianchi, C., Kerr, G., \& Patti, C. (2010). "Consumer-based brand equity for Australia as a long-haul tourism destination in an emerging market". International Marketing Review , 27 (4), 434-449.

Pitta, D. A., \& Katsanis, L. P. (1995). "Understanding brand equity for successfull brand extension". Journal of Consumer Marketing , 12 (4), 51-64.

Policy, A. D. Automotive Developement Policy (ADP) 2016-21.

Prabha, A. (., Savard, K., \& Wickramarachi, H. (March de 2014). Deriving the Economic Impact of Derivatives. Milken Institute .

Program, A. I. (2008). Ministry of Industries, Production and Special Initiatives, Government of Pakistan.

Sundaram, R. K. (September de 2012). Derivatives in Financial Market Development. Working paper. International Growth Centre.

Sajjad, F., Noreen, U., \& Zaman, K. (2013). Impact of Derivatives on Financial Services Sector and Risk Management. Middle-East Journal of Scientific Research , 18 (6), 748-758.

Saroha, D. P., \& Yadav, D. S. (2013). Derivative market in India: Prospects \& Issues. International Multidiciplenery e-Journal . 
Schau, H. J., \& Gilly, M. C. (2003). "We are what we post? Self-presentation in personal web space". Journal of Consumer Research, 30 (3), 385-404.

Schivinski, B., \& Dabrowski, D. (2015). "The impact of brand communications on brand equity through Facebook". Journal of Research in Interactive Marketing , 9 (1), 31-53.

Seitz, V., Razzouk, N., \& Wells, D. M. (2010). " The importance of brand equity on purchasing consumer durables: an analysis of home air-conditioning systems. Journal of Consumer Marketing , 27 (3), 236-242.

Sill, K. (1997). The Economic Benefits and Risks Of Derivative Securities. Business Review . FEDERAL RESERVE BANK OF PHILADELPHIA.

Small \& Mediun Enterprise Developement Authority, G. o. (2011). SME Developement Report (2010-2011), Programmes \& Initiatives. Lahore: Small \& Mediun Enterprise Developement Authority, Governmrnt of Pakistan.

SMEDA, IMC, Suzuki, P., \& Toyota. (2012 - 2016). Annual Reports Automobile Companies.

Smith, A. N., Fischer, E., \& Yongjian, C. (2012). "How does brand-related user-generated content differ across YouTube, Facebook and Twitter?". Journal of Interactive Marketing , 26 (2), 102-113.

Soler Pujals, P. (2001). Investigación de mercados. Barcelona, España: Universidad Autónoma de Barcelona.

Srivastava, D. A. (September de 2010). Derivative market in India status and prospects .

Steenkamp, J. E., \& Geyskens, I. (2006). "How country characteristics affect the perceived value of a website". Journal of Marketing , 70, 136-150.

R.Turner, E. (2017). Travel\&Tourism Economic Impact 2017 Ghana. World Travel\& Toursim Council .

Rahman, S., \& Hassan, M. K. (2011). The Potential of Derivatives Market in Bangladesh. Journal of Economic Cooperation and Development, 32 (4), 97-144.

Rao, A. R., \& Monroe, K. B. (1989). "The effect of price, brand name and store name on buyers' perception of product quaility: an integrative review". Journal of Marketing Research, 36 (2), 351-358.

Renó, D., Gosciola, V., \& Renó, L. (2018). Nova Ecologia dos Meios e Tecnologia. Ria Editorial - Comité Cientifico.

Research, C. o. (January de 2017). Trade benefits to the automotive industry and potential consequences of withdrawal from the agreement. NAFTA BRIEFING , pp. 1-19.

Tourism, M. o. (2012). National Tourism Development Plan(2013-2027). http://www.ghana.travel/wp-content/uploads/2016/1.

Treviño, L. (2005). Development and volume growth of organized derivatives trade in emerging markets. Ensayos , XXIV (2) , 31-82. 
Anexos

Anexo \#1 Desigualdad por ingresos - Coeficiente de GINI (INEC)

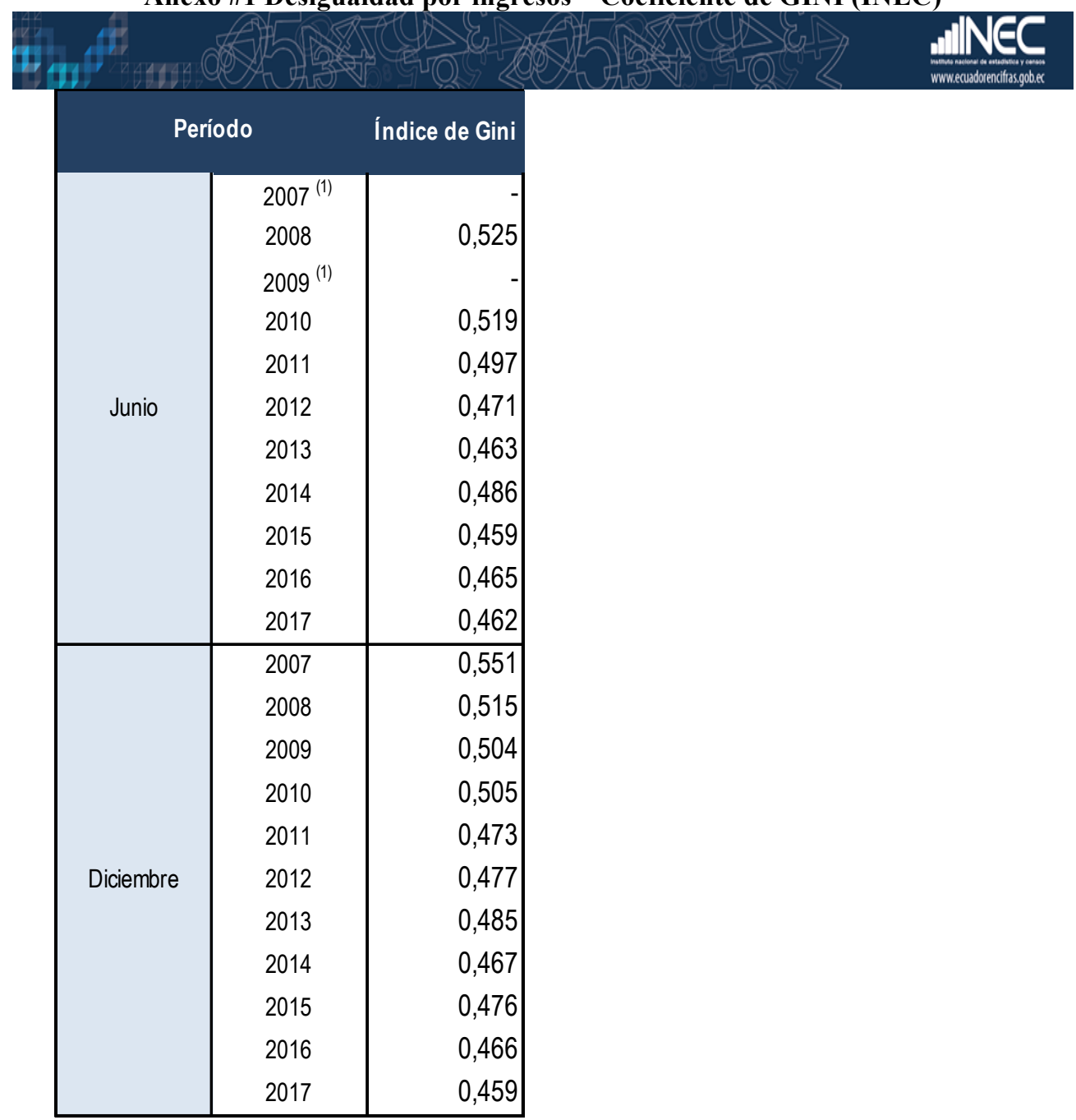

Fuente: Encuesta Nacional de Empleo, Desempleo y Subempleo - ENEMDU.

Notas: (1) La información en junio 2007 y junio 2009 solo fue representativa a nivel urbano. 
Anexo \#2 Porcentaje de personas analfabetas digitales (INEC)

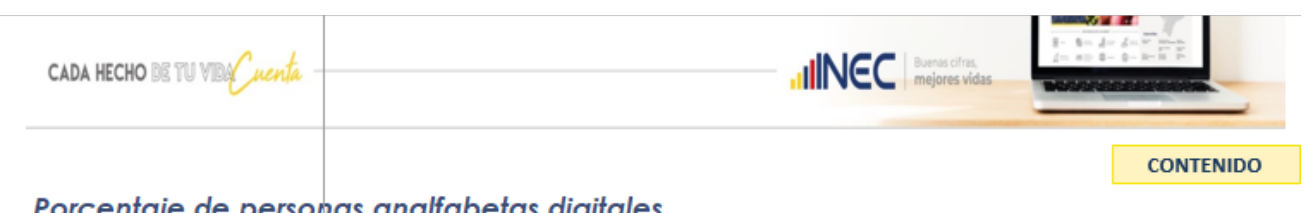

\begin{tabular}{|c|c|c|c|c|c|c|c|}
\hline Periodo & Nacional & $\frac{8}{2}$ & Urbana & Rural & ఖ & Hombre & Mujer \\
\hline dic-08 & 32,42 & & 22,95 & 53,81 & & 28,61 & 35,95 \\
\hline dic-09 & 33,60 & & 25,34 & 52,32 & & 30,23 & 36,81 \\
\hline dic- 10 & 29,22 & & 20,83 & 48,02 & & 26,28 & 32,07 \\
\hline dic-11 & 25,14 & & 17,68 & 42,37 & & 22,17 & 27,91 \\
\hline dic- 12 & 21,38 & & 15,07 & 35,62 & & 18,97 & 23,70 \\
\hline dic- 13 & 20,04 & & 15,47 & 30,57 & & 18,13 & 21,89 \\
\hline dic-14 & 14,43 & & 9,80 & 25,01 & & 12,02 & 16,70 \\
\hline dic- 15 & 12,22 & & 7,39 & 23,48 & & 10,09 & 14,24 \\
\hline dic-16 & 11,45 & & 6,85 & 21,99 & & 9,37 & 13,45 \\
\hline dic- 17 & 10,48 & & 5,99 & 21,24 & & 8,92 & 11,96 \\
\hline dic-18 & 10,68 & & 7,17 & 18,98 & & 9,61 & 11,72 \\
\hline dic-19 & 11,39 & & 7,80 & 20,05 & & 10,63 & 12,13 \\
\hline
\end{tabular}

Fuente: Encuesta Nacional Multipropósito de Hogares, diciembre 2018 y 2019.

Elaboracion: Instituto Nacional de Estadística y Censos. INEC

Notas:

- Las cifros anteriores al 2018 son fuente: Encuesta Nacional de Empleo, Desempleo y Subempleo (ENEMDU).

Se considera Analfabeta Digital a ung persono de 15049 años cuando cumple simultóneamente tres características: 1) No tiene celular activado 2) En los últimos 12 meses no ha utilizado computadora 3) En los últimos 12 meses no ha utilizado Intemet.

Población de referencia La población en edad 15 a 49 años

- Los datos se presentan en puntos porcentuales 